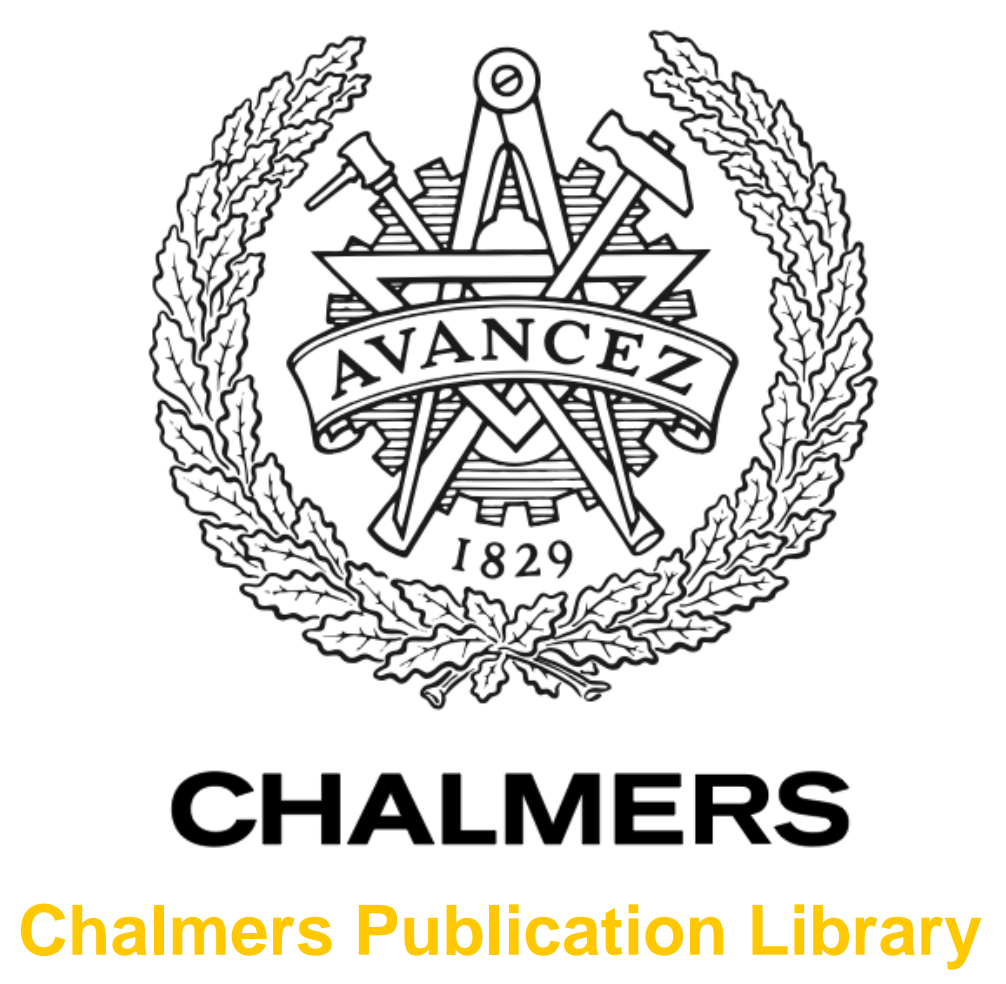

\title{
Simple calculation of ergodic capacity of lossless two-port antenna system using only S-parameters - Comparison with common Z-parameter approach
}

This document has been downloaded from Chalmers Publication Library (CPL). It is the author's version of a work that was accepted for publication in:

2011 IEEE International Symposium on Antennas and Propagation, Spokane, USA, 3-8 July, 2011 (ISSN: 1522-3965)

Citation for the published paper:

Chen, X. ; Kildal, P. ; Carlsson, J. (2011) "Simple calculation of ergodic capacity of lossless two-port antenna system using only S-parameters - Comparison with common Z-parameter approach". 2011 IEEE International Symposium on Antennas and Propagation, Spokane, USA, 3-8 July, 2011 pp. 1885-1888.

http://dx.doi.org/10.1109/APS.2011.5996867

Downloaded from: http://publications.lib.chalmers.se/publication/143612

Notice: Changes introduced as a result of publishing processes such as copy-editing and formatting may not be reflected in this document. For a definitive version of this work, please refer to the published source. Please note that access to the published version might require a subscription.

Chalmers Publication Library (CPL) offers the possibility of retrieving research publications produced at Chalmers University of Technology. It covers all types of publications: articles, dissertations, licentiate theses, masters theses, conference papers, reports etc. Since 2006 it is the official tool for Chalmers official publication statistics. To ensure that Chalmers research results are disseminated as widely as possible, an Open Access Policy has been adopted.

The CPL service is administrated and maintained by Chalmers Library. 


\section{Simple Calculation of Ergodic Capacity of Lossless Two-Port Antenna System Using Only S-Parameters - Comparison with Common Z-parameter Approach}

\author{
Xiaoming Chen, Per-Simon Kildal \\ Department of Signals and Systems \\ Chalmers University of Technology \\ Gothenburg, Sweden \\ xiaoming.chen@chalmers.se
}

\author{
Jan Carlsson \\ Electronics department \\ SP Technical Research Institute of Sweden \\ Boras, Sweden
}

\begin{abstract}
In this paper, we show how to correctly include overall antenna effects in a numerically generated Rayleigh channel matrix when calculating ergodic MIMO capacity of lossless multi-port antenna system located in rich isotropic multipath. The overall antenna effects can be included correctly using either impedance parameters (Z-parameter method) or scattering parameters (S-parameter method). These two methods are compared and validated by measurements in anechoic and reverberation chambers. A narrowband handset antenna with negligible ohmic loss is used for the measurements. There are very good agreements between the capacities obtained using both methods from anechoic chamber measurement and that from direct channel measurement in reverberation chamber.
\end{abstract}

Keywords-MIMO capacity, reverberation chamber, anechoic chamber.

\section{INTRODUCTION}

Multi-port antenna systems have shown substantial increase in attainable data rate in multipath environments [1]-[3]. In early literature, antenna elements in multi-port antenna system were considered isotropic and lossless. The effect of correlated fading at antenna ports was studied in this way in [2] and [4], and it was shown that correlation reduced the ergodic MIMO capacity. The overall antenna effects were examined in [3] and [5]-[7] via impedance parameter of the multi-port antenna (Zparameter method). The method requires a priori knowledge of open-circuit correlation of the multi-port antennas, which is difficult to measure in practice. There exist other ways of including overall antenna effects when evaluating capacity [8]-[10]. Despite of different forms, all the above mentioned methods can correctly take overall antenna effects into account. It is well known that the free space S-parameters of a two-port antenna can be used to determine the correlation and diversity gain [9], [10]. Herein, based on similar methodology as in Z-parameter meter, we propose to use the S-parameters and a numerically generated fading channel to determine also capacity.

Need to mention that both Z-parameter and S-parameter methods work only for lossless antennas. It has been shown

This work has been supported in part by The Swedish Governmental Agency for Innovation Systems (VINNOVA) within the VINN Excellence Center Chase. that for lossy wideband antenna, the channel measurements in reverberation chamber and embedded far field functions and efficiencies measured in anechoic chamber render the same capacities over all frequencies [11]. In this paper, a narrowband handset antenna with negligible ohmic loss is used. We use Sparameter and Z-parameter methods to calculate MIMO capacity based on S-parameters measured in anechoic chamber. It has been shown that ergodic MIMO capacity can be measured readily in reverberation chamber [9], [10]. The Sparameter method is first validated with reverberation chamber measurement. Excellent agreement is observed. This is of particular interest because both reverberation chambers and anechoic chambers are being considered for standardization of so-called Over-The-Air (OTA) measurements for characterization of active MIMO stations. The S-parameter method then is compared with Z-parameter method, good agreement is again observed. It is shown that, compared with Z-parameter method, S-parameter method is easier to use for practical antenna.

\section{MIMO CAPACITY}

We assume the receiver has perfect channel state information (CSI), and that transmitted power is equally allocated among transmitting antenna elements. The maximum available capacity of the multi-port antenna system can then be expressed as [1]

$$
C_{N_{r} \times N_{t}}=E\left\{\log _{2}\left[\operatorname{det}\left(\mathbf{I}_{N_{r}}+\frac{\gamma}{N_{t}} \mathbf{H}_{N_{r} \times N_{t}} \mathbf{H}_{N_{r} \times N_{t}}^{H}\right)\right]\right\}
$$

where $\gamma$ is signal-to-noise ratio (SNR), the superscript $H$ is Hermitian operator, $\mathbf{H}_{N_{r} \times N_{t}}$ is the channel matrix, $N_{t}$ and $N_{r}$ are number of transmitting and receiving antennas respectively, and $E$ is statistical expectation taken over samples of the channel matrix describing the fading. The subscripts in (1) will be dropped hereafter for conciseness. $\mathbf{H}$ is normalized so that its Frobenius norm satisfies $E\left[\|\mathbf{H}\|_{F}^{2}\right]=N_{t} N_{r}$.

In this paper, we focus on compact power-balanced twoport antennas in receive end of the MIMO systems. Therefore, 
it is judicious to assume ideal transmitting antennas with unity efficiency and no correlation. Furthermore, since a lossless antenna is used, both S-parameter method and Z-parameter method can be used based on anechoic chamber measurement.

\section{A. Z-Parameter Method}

The overall antenna effects can be included using Zparameter method [5]-[7] by assuming lossless multi-port antenna in single mode operation. The open circuit voltages on the receiving MIMO array are related to the voltages at the output ports when the ports are terminated in the diagonal load matrix $\mathbf{Z}_{L}$ by [5]-[7]

$$
\begin{aligned}
& \mathbf{V}_{\mathbf{r}}=\mathbf{C}_{\mathbf{r}} \mathbf{V}^{\mathbf{o c}} \\
& \mathbf{C}_{\mathbf{r}}=\mathbf{Z}_{L}\left[\mathbf{Z}+\mathbf{Z}_{L}\right]^{-1}
\end{aligned}
$$

By assuming complex conjugate of self-impedance, $\mathbf{C}_{\mathbf{r}}$ reduces to $\left(Z_{L}+Z_{11}\right)\left[\mathbf{Z}+\mathbf{Z}_{L}\right]^{-1}$ [5]. In practice, antenna impedance cannot be measured directly. Therefore the antenna impedance matrix $\mathbf{Z}$ has to be converted from measured $\mathbf{S}$ parameters by [14]

$$
\begin{aligned}
& Z_{11}=Z_{0} \frac{\left(1+S_{11}\right)\left(1-S_{22}\right)+S_{12} S_{21}}{\left(1-S_{11}\right)\left(1-S_{22}\right)-S_{12} S_{21}} \\
& Z_{12}=Z_{0} \frac{2 S_{12}}{\left(1-S_{11}\right)\left(1-S_{22}\right)-S_{12} S_{21}} \\
& Z_{21}=Z_{0} \frac{2 S_{21}}{\left(1-S_{11}\right)\left(1-S_{22}\right)-S_{12} S_{21}} \\
& Z_{22}=Z_{0} \frac{\left(1-S_{11}\right)\left(1+S_{22}\right)+S_{12} S_{21}}{\left(1-S_{11}\right)\left(1-S_{22}\right)-S_{12} S_{21}}
\end{aligned}
$$

The correlated channel including mutual coupling effect is [7]

$$
\mathbf{H}_{Z}^{m c}=2 \sqrt{R_{11}} \mathbf{R}_{L}^{1 / 2}\left[\mathbf{Z}+\mathbf{Z}_{L}\right]^{-1} \mathbf{K}_{\mathbf{r}}^{o c} \mathbf{H}
$$

where $R_{11}$ and $\mathbf{R}_{L}$ are the real parts of $Z_{11}$ and $\mathbf{Z}_{L}$ respectively, $\mathbf{H}$ is uncorrelated numerically generated channel matrix, and $\mathbf{K}_{\mathrm{r}}^{\text {oc }}$ is Cholesky decomposition of opencircuit correlation matrix $\boldsymbol{\Phi}_{\mathrm{r}}^{\mathrm{oc}}$. In practice, the open-circuit correlation of a multi-port antenna can be difficult to determine. But for electrically small antenna in threedimensional (3-D) isotropic scattering environments, it can be well approximated by [12], [13]

$$
\begin{aligned}
& \boldsymbol{\Phi}_{r}^{o c}=\left[\begin{array}{cc}
1 & \rho^{o c} \\
\rho^{o c} * & 1
\end{array}\right] \\
& \rho^{o c}=\frac{\operatorname{Re}\left\{Z_{12}\right\}}{\sqrt{\operatorname{Re}\left\{Z_{11}\right\} \operatorname{Re}\left\{Z_{22}\right\}}}
\end{aligned}
$$

The MIMO capacity including overall antenna effects is

$$
C=E\left\{\log _{2}\left[\operatorname{det}\left(\mathbf{I}+\frac{\gamma}{N_{t}} \mathbf{H}_{Z}^{m c}\left(\mathbf{H}_{Z}^{m c}\right)^{H}\right)\right]\right\}
$$

\section{B. S-Parameter Method}

Instead of using Z-parameter method mentioned above, we found it is more convenient to treat open-circuit correlation and mutual coupling together as actual correlation in practice. The correlated channel matrix can then be expressed as

$$
\mathbf{H}_{S}^{m c}=\mathbf{K}_{\mathbf{r}}^{\mathbf{m c}} \mathbf{H}
$$

where $\boldsymbol{\Phi}_{\mathbf{r}}^{\mathbf{m c}}=\mathbf{K}_{\mathbf{r}}^{\mathbf{m c}}\left(\mathbf{K}_{\mathbf{r}}^{\mathbf{m c}}\right)^{H}$ is the actual correlation matrix, which can be readily measured in practice. When the ohmic loss in the antenna is negligible, the total embedded radiation efficiency including mismatch factor is defined by [15]

$$
e_{e m b}=1-\left|S_{11}\right|^{2}-\left|S_{21}\right|^{2}
$$

where $S_{11}$ and $S_{21}$ are the S-parameters of the antenna in free space. The correlation matrix is

$$
\boldsymbol{\Phi}_{\mathbf{r}}^{m c}=\left[\begin{array}{cc}
1 & \rho \\
\rho^{*} & 1
\end{array}\right]
$$

In isotropic scattering environment, the complex correlation (with mutual coupling effects included) is [16]

$$
\rho=\frac{-\left(S_{11}^{*} S_{12}+S_{21}^{*} S_{22}\right)}{\sqrt{\left(1-\left|S_{11}\right|^{2}-\left|S_{21}\right|^{2}\right)\left(1-\left|S_{22}\right|^{2}-\left|S_{21}\right|^{2}\right)}}
$$

The MIMO capacity is

$$
C=E\left\{\log _{2}\left[\operatorname{det}\left(\mathbf{I}+\frac{\gamma e_{e m b}}{N_{t}} \mathbf{H}_{S}^{m c}\left(\mathbf{H}_{S}^{m c}\right)^{H}\right)\right]\right\}
$$

Equation (11) shows the effects of embedded radiation efficiency and correlation on capacity explicitly.

\section{MEASUREMENTS}

Ergodic MIMO capacity for lossless antennas can be calculated using both methods mentioned above based on antenna S-parameter measurement in anechoic chamber.

The Bluetest HP reverberation chamber in use is shown in Fig. 1. It has a size of $1.75 \mathrm{~m} \times 1.25 \mathrm{~m} \times 1.8 \mathrm{~m}$ and is provided with two plate stirrers, and platform and polarization stirring [10]. In the measurements, the platform was moved to 20 positions spaced by $18^{\circ}$, and for each platform position each of the two plates move simultaneously to 10 positions, evenly distributed along the total distance they can move. At each stirrer position and for each of the three wall antennas, a full frequency sweep was performed by the vector network analyzer. A well stirred reverberation chamber emulates a rich isotropic multipath environment [10]. It has been shown that ergodic MIMO capacity can be determined easily from reverberation chamber measurement [9]. The measurements were performed as follows: First, the channel transfer function is measured using a reference antenna with known efficiency. The power transfer function averaged over all the stirring samples gives the reference level, $P_{\text {ref, }}$, with the reference antenna efficiency calibrated out. Thereafter, the antenna under test was measured, giving the channel matrix $\mathbf{H}_{2 \times 3}$ as a function of frequency and stirrer positions. The reverberation chamber has three wall antennas, so we chose to evaluate a $2 \times 3$ MIMO system from the measured data. Of convenience, we introduce the following notation for the normalized measured channel matrix

$$
\mathbf{H}_{\text {meas }}=\mathbf{H}_{2 \times 3} / \sqrt{P_{\text {ref }}}
$$


The MIMO capacity can then be computed from the measured channel matrices by using

$$
C=E\left\{\log _{2}\left[\operatorname{det}\left(I+\frac{\gamma}{N_{t}} \mathbf{H}_{\text {meas }} \mathbf{H}_{\text {meas }}^{H}\right)\right]\right\}
$$

Note that the reverberation chamber attenuation and the total radiation efficiency of the wall antennas are calibrated out by (12). Since the three wall antennas in the reverberation chamber are located far away from each other on three orthogonal walls, the correlations between them are negligible.

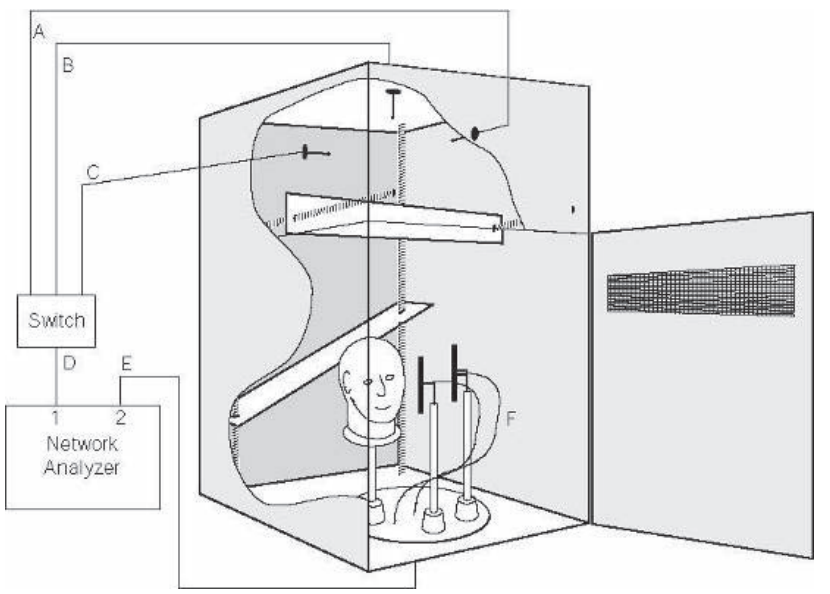

Fig. 1. Drawing of Bluetest reverberation chamber with two mechanical plate stirrers, platform, three wall antennas and example of two-port antenna under test.

The narrowband portable two-port antenna (see Fig. 2) has negligible ohmic loss (working at $1.6 \mathrm{GHz}$ ) [18]. Therefore, we choose S-parameter method to calculate the MIMO capacity based on S-parameters measured in anechoic chamber. In order to see the effects of total embedded radiation efficiency and correlation on capacity, we plot in Fig. 3 the $2 \times 3$ MIMO capacity for the following four cases: assuming $100 \%$ efficiency and zero correlation; including the measured efficiency; and including both efficiency and correlation; and the MIMO capacity obtained from the measured channel matrix in the reverberation chamber using (13). We see that the total embedded radiation efficiency reduces the capacity, and that the correlation (with mutual coupling presented) reduces it further, and that the $\mathrm{S}$ parameter method gives MIMO capacity in excellent agreement with the values from measurements in reverberation chamber.

Fig. 4 compares calculated capacities using Z-parameter and S-parameter methods. From Fig. 4, it can be seen that both methods give similar results. The little bias between them is probably due to the approximation of open-circuit correlation of (5) using Z-parameter method, which is true only for minimum scattering antennas [13].

Note that neither Z-parameter method nor S-parameter method is valid for general lossy multi-port antennas, while MIMO channels measured in reverberation are generally valid for any multi-port antenna [11].

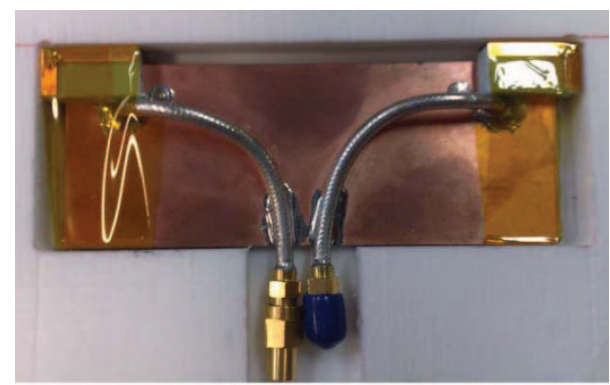

Fig. 2. Photo of the two-element portable antenna used for verifying Sparameter method.

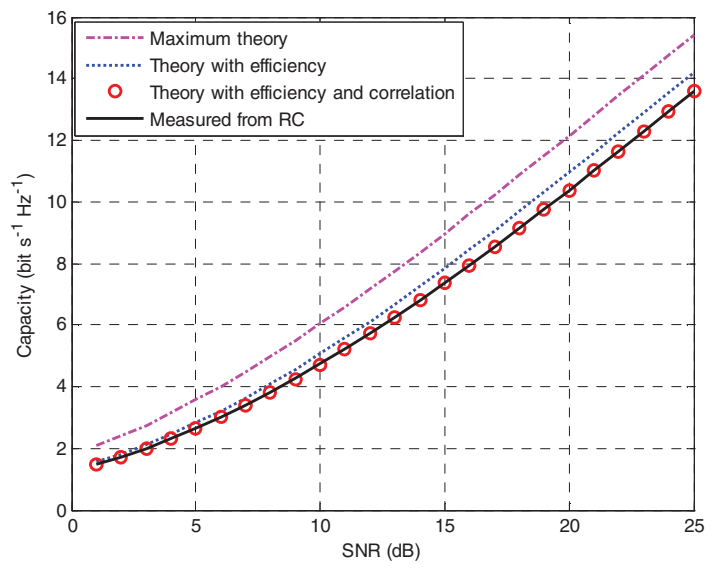

Fig. 3. $2 \times 3$ MIMO capacity. The curves show the degradation from the theoretical maximum due to embedded element efficiency and correlation, evaluated using S-parameter method, and agreement with capacity based on measured channel matrix in reverberation chamber.

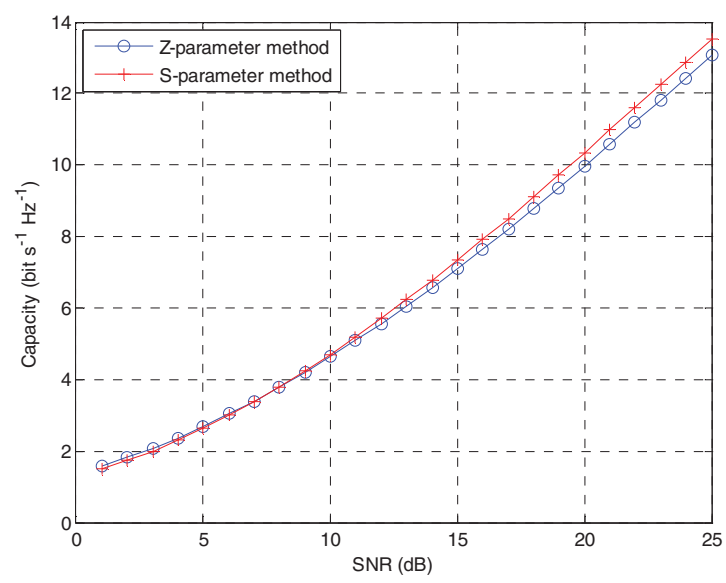

Fig. 4. 2×3 MIMO capacity using Z-parameter and S-parameter methods.

\section{CONCLUSION}

In this paper we have compared two methods to calculate MIMO capacity of a MIMO system with lossless two-port receive antenna based on anechoic chamber measurement. For lossless antennas, both Z-parameter method and S-parameter can be used, and Z-parameter method agrees with S-parameter for electrically small antennas, which can be approximated by 
minimum scattering antennas. Moreover, S-parameter is easier to use for practical antenna. Comparison between ergodic capacities based on anechoic and reverberation chamber measurements show excellent agreement. Reverberation chamber is much easier for MIMO capacity measurement in the sense that it does not involve any channel modeling, since MIMO channel matrix can be directly measured in reverberation chamber. While both S-parameter and Zparameter methods are restricted to lossless antennas, channelmeasurement based reverberation chamber measurement is valid for any multi-port antenna.

\section{REFERENCES}

[1] G. J. Foschini and M. J. Gans, "On limits of wireless communications in a fading environment when using multiple antennas," Wireless Personal Communications, vol.6, no. 3, pp. 311-335, Mar. 1998.

[2] B. Vucetic and J. Yuan, Space-time coding, Wiley, 2003.

[3] C. Oestges and B. Clerckx, MIMO wireless communications: from realworld propagation to space-time code design. Academic Press, 2007.

[4] D. Chizhik, F. Farrokhi, J. Ling and A. Lozano, "Effect of antenna separation on the capacity of BLAST in correlated channels," IEEE Communication Letters, vol. 4, no. 11, pp. 337-339, Nov. 2000.

[5] R. Janaswamy, "Effect of element mutual coupling on the capacity of fixed length linear arrays," IEEE Antennas and Wireless Propag. Lett., vol. 1, pp. 157-160, 2002.

[6] W. F. Tsen and H. Li, "Uncoupled impedance matching for capacity maximization of compact MIMO arrays", IEEE Antennas and Wireless Propag. Lett., vol. 8, pp. 1295-1298, 2009.

[7] Y. Fei, Y. Fan, B. K. Lau and J. S. Thompson, "Optimal single-port matching impedance for capacity maximization in compact MIMO arrays", IEEE Trans. Antennas and Propag., vol. 56, no. 11, pp. 35663575, Nov. 2008.

[8] J. W. Wallance and M. A. Jensen, "Mutual coupling in MIMO wireless systems: a rigorous network theory analysis", IEEE Trans. Wireless Commun., vol. 3, no. 4, pp. 1317-1325, July 2004.

[9] K. Rosengren and P-S. Kildal, "Radiation Efficiency, Correlation,
Diversity Gain and Capacity of a Six Monopole Antenna Array for a MIMO System: Theory, Simulation and Measurement in Reverberation Chamber," in Proc. IEE, Microwaves, Optics and Antennas, pp. 7-16, Vol.152, No.1, Feb 2005. See also Erratum published in August 2006.

[10] P.-S. Kildal and K. Rosengren, "Correlation and capacity of MIMO systems and mutual coupling, radiation efficiency and diversity gain of their antennas: Simulations and measurements in reverberation chamber", IEEE Communications Magazine, vol. 42, no. 12, pp. 102 112, Dec. 2004.

[11] X. Chen, P.-S. Kildal, J. Carlsson and J. Yang, "Comparison of ergodic capacities from wideband MIMO antenna measurements in reverberation chamber and anechoic chamber", submitted to IEEE Antennas and Wireless Propagat. Lett., Feb. 2011.

[12] R. G. Vaughan and J. B. Andersen, "Antenna diversity in mobile communications", IEEE Trans. Vehic. Technol. vol. 36, no. 4, pp. 149172, Nov. 1987.

[13] W. Wasylkiwskyj and W. K. Kahn, "theory of mutual coupling among minimum-scattering antennas", IEEE Trans. Antennas and Propag., vol. 18, no. 2, pp. 204-216, Mar., 1970.

[14] D. M. Pozar, Microwave engineering, 3rd edition, John Wiley \& Sons, 2005.

[15] M. Ng Mou Kehn, M. V. Ivashina, P.-S. Kildal and R. Maaskant, "Definition of unifying decoupling efficiency of different array antennas - case study of dense focal plane array feed for parabolic reflector", AEUE-International Journal of Electronics and Com-munications, 2009 - Elsevier, available on line 4 June 2009.

[16] S. Blanch, J. Romeu and I. Corbella, "Exact representation of antenna system diversity performance from input parameter description", Electronics letters, vol. 39, no. 9, pp. 705-707, May 2003.

[17] R. G. Vaughan and J. B. Andersen, "Antenna diversity in mobile communications", IEEE Trans. Vehic. Technol. vol. 36, no. 4, pp. 149172, Nov. 1987.

[18] A.H. Azremi, J. Toivanen, T. Laitinen, P. Vainikainen, X. Chen, N. Jamaly, J. Carlsson, P.-S Kildal, S. Pivnenk, "On Diversity Performance of Two-Element Coupling Element Based Antenna Structure for Mobile Terminal", EuCAP 2010 Fourth European Conference on Antennas and Propagation, Barcelona, Spain, 2010. 